\title{
BMJ Open Serum alkaline phosphatase and the risk of coronary heart disease, stroke and all-cause mortality: Tehran Lipid and Glucose Study
}

\author{
Maryam Kabootari, ${ }^{1,2}$ Mohammad Reza Raee, ${ }^{3}$ Samaneh Akbarpour, ${ }^{4}$ \\ Samaneh Asgari, ${ }^{2}$ Fereidoun Azizi, ${ }^{5}$ Farzad Hadaegh ${ }^{2}$
}

To cite: Kabootari M, Raee MR, Akbarpour S, et al. Serum alkaline phosphatase and the risk of coronary heart disease, stroke and all-cause mortality: Tehran Lipid and Glucose Study. BMJ Open 2018;8:e023735. doi:10.1136/ bmjopen-2018-023735

- Prepublication history and additional material for this paper are available online. To view these files, please visit the journal online (http://dx.doi org/10.1136/bmjopen-2018023735).

Received 28 April 2018 Revised 11 August 2018 Accepted 5 October 2018
Check for updates

(C) Author(s) (or their employer(s)) 2018. Re-use permitted under CC BY-NC. No commercial re-use. See rights and permissions. Published by BMJ.

For numbered affiliations see end of article.

Correspondence to Dr Farzad Hadaegh; fzhadaegh@endocrine.ac.ir

\section{ABSTRACT}

Objectives To investigate the association of alkaline phosphatase (ALP) levels with the risk of the composite end point of cardiovascular disease (CVD), and all-cause mortality as well as each of them separately.

Design Prospective cohort study.

Setting Within the framework of the Tehran Lipid and Glucose Study (TLGS) cohort, participants were followed from baseline examination (1999-2001) until March 2014. Participants A total of 2578 participants, aged $\geq 30$ years free of prevalent CVD at baseline examination.

Primary outcome The main outcome measures were composite end point of coronary heart disease (CHD), stroke, all-cause mortality and each per se.

Results During a median follow-up of 11.3 years, 369 , 68, 420, 170 and 495 participants experienced CHD, stroke, CVD, all-cause mortality and the composite outcome, respectively. In the multivariable Cox regression models, the adjusted HRs $(95 \% \mathrm{Cl})$ for mentioned events per one SD increase in ALP level after full adjustment were 1.11 (1.01 to 1.22$), 1.20$ (0.97 to $1.49, p=0.058), 1.10$ (1.01 to 1.21), 1.16 (1.01 to 1.33) and 1.11 (1.02 to 1.21), respectively. Furthermore, participants with ALP levels in the highest tertile had significant adjusted $\mathrm{HRs}(95 \% \mathrm{Cl})$ for stroke (1.88 (1.00 to 3.61)), CVD (1.30 (1.01 to 1.68)) and composite outcome (1.27 (1.00 to 1.61)). The cut-off value of $A L P \geq 199 \mathrm{IU} / \mathrm{L}$ for predicting composite outcome was derived using Youden's index, based on which this cut-off point was associated with significant risk of $80 \%, 26 \%$, $43 \%$ and $26 \%$ for incident stroke, CVD, all-cause mortality and composite outcome. Additionally, no improvement was seen in the predictive ability of traditional risk factors models after adding ALP values, considering the levels of Akaike information criterion, C-index and Net Reclassification Index.

Conclusion Independent associations between ALP levels and the risks of CVD and mortality events were shown, despite the fact that adding the data of ALP to known risk factors did not improve the prediction of these events.

\section{INTRODUCTION}

Heart disease-related mortality is the leading cause of death in the USA and worldwide. ${ }^{12}$ According to our previous study, the

\section{Strengths and limitations of this study}

- This study is a a population-based cohort with large sample size and a long-term follow-up years among general population.

- The study evaluated different covariates with reliable methods and assessed different outcomes including coronary heart disease, stroke, cardiovascular disease (CVD) and all-cause mortality.

- This study is the first community-based prospective cohort in a Middle Eastern population with high incidence and burden of CVD that assessed the association between alkaline phosphatase (ALP) level and different outcomes.

- Information regarding some confounding factors including Parathyroid Hormone (PTH), C reactive protein, vitamin $\mathrm{D}$ and liver enzymes were not available in the database; these factors could affect the association between ALP and different outcomes.

incidence of coronary heart disease (CHD) in an Iranian population is comparable with that of the peak years of CHD incidence (1971-1989) in the USA. ${ }^{3}$ In addition, over $40 \%$ of deaths among Iranian population are related to cardiovascular disease (CVD), approximately $20 \%$ of adults aged $\geq 30$ years had symptoms or signs of $\mathrm{CHD}^{4}$ and over $70 \%$ of them had at least one CHD risk factor. ${ }^{5}$

There is increasing evidence that coagulation and inflammatory factors play major roles in the pathogenesis of CVD by initiating and facilitating the development of atherothrombotic mechanisms. ${ }^{6}$ During the past decade, serum alkaline phosphatase (ALP), an inflammatory marker, ${ }^{7}$ has emerged as a novel marker for the risk of CVD in the general population in several studies. ${ }^{8-10}$ Recently, results of two meta-analyses conducted by Kunutsor et al derived from population-based studies conducted in the USA, Europe and Japan showed that every one SD (1-SD) increase in baseline ALP was 
associated with $8 \%$ higher risk for incident CVD. Moreover, when compared with the bottom third, levels in the top third of ALP were associated with $38 \%$ higher risk of all-cause mortality. ${ }^{11}{ }^{12}$ However, only one study has examined the added value of ALP over traditional risk factors for incidence of CVD, ${ }^{8}$ while no study has examined this issue for mortality events.

The aim of this study was to assess the association of serum ALP with incident CHD, stroke, CVD, all-cause mortality and composite outcome (including CVD and mortality events), independent of traditional risk factors, in a Middle Eastern population with high incidence and burden of $\mathrm{CVD},{ }^{313}$ and also, if such an association exists, to determine the extent to which ALP can improve our power to predict CVD and mortality events beyond that achieved by traditional risk factors. ${ }^{14} 15$

\section{MATERIALS AND METHODS}

\section{Study population}

The Tehran Lipid and Glucose Study (TLGS) is a population-based prospective study being conducted on a representative sample of residents of district 13 of Tehran, the capital city of Iran. Details and data collection methods of TLGS have been described elsewhere. ${ }^{16}$ To summarise, TLGS has two major components: a cross-sectional prevalence study of non-communicable disease (1999-2001) and associated risk factors; and prospective follow-up studies at approximately 3-year intervals. For the current study, we considered the data of 3359 participants, aged $\geq 30$ years, who had documented measurements of ALP level at baseline. Excluded were individuals with a history of CVD $(n=251)$ and cancer $(\mathrm{n}=108)$, those with missing data of covariates (105 participants) and those without any follow-up after the baseline recruitment (317 participants), leaving 2578 adults with complete follow-up data up to March 2014. The study was conducted according to the guidelines of the Declaration of Helsinki. Written informed consents were obtained from all participants.

\section{Patient and public involvement}

Participants were not involved in setting of the research agenda.

\section{Data collection (clinical, anthropometric and laboratory measurements)}

Participants were interviewed face-to-face by trained interviewers using a standard questionnaire, including information on gender, age, smoking status, family history of premature CVD and medication use. A standardised mercury sphygmomanometer (calibrated by Iranian Institute of Standards and Industrial Researches) was used to measure blood pressure after the participants had been seated for at least $15 \mathrm{~min}$. The mean of two measurements of blood pressures taken on the right arm was considered the participant's blood pressure. Details of anthropometric measures including height and waist circumference (WC) have been reported elsewhere. ${ }^{16}$ Body mass index (BMI) was calculated by dividing weight in kilograms by height in square metres. After 12-14hours of overnight fasting, a blood sample was drawn from all participants. Details of laboratory measurements including levels of fasting plasma glucose (FPG), triglycerides (TG), high-density lipoprotein cholesterol (HDL-C) and total cholesterol (TC) and serum creatinine have been reported previously. ${ }^{16}$ Serum ALP was measured using the DGKC (Deutsche Gesells chaftfür Klinische Chemie) method (upper limit of normal=306 IU/L; intra-assay coefficient of variation $=1.5 \%$ ).

\section{Outcome assessment}

Details of collection of TLGS outcome data have been published before ${ }^{17}$ Briefly, telephone follow-up of each participant for any new medical events was performed annually by a trained nurse. Then, a trained physician collected complementary data regarding reported events during a home visit or by obtaining data from medical records. An outcome committee consisting of an internist, an endocrinologist, a cardiologist, an epidemiologist and other experts, when needed, evaluated the collected data to assign a specific outcome code to each event. In this study, CHD events as outcomes included cases of definite myocardial infarction (MI) (diagnosed by ECG and biomarkers), probable MI (positive ECG findings plus cardiac symptoms or signs and biomarkers showing negative or equivocal results), unstable angina pectoris (new cardiac symptoms or changing symptom patterns and positive ECG findings with normal biomarkers), angiography-proven CHD and CHD death (any death in hospital due to CHD according to the above-mentioned criteria or sudden cardiac death caused by cardiac disease occurring $\leq 1$ hour after beginning of symptoms based on verbal autopsy documents outside of hospital). Moreover, stroke was defined as cases of definite stroke, possible stroke and transient ischaemic attack (TIA) with the following definitions: (1) Definite stroke was defined based on the WHO definition as, 'rapidly developing clinical signs of or global disturbance of cerebral function, lasting $>24$ hour or leading to death with no apparent cause other than that of vascular origin ${ }^{18}$ or imaging result suggestive of stroke accompanied by acute clinically relevant brain injuries in patients with fast vanishing symptoms. (2) Possible stroke was considered as onset of an acute focal neurological deficit without imaging indicative of stroke or when data did not fully qualify for the WHO definition of definite stroke. (3) When symptoms resolved within 24 hours, patients were considered as cases with TIA.

CVD was specified as a composite measure of any CHD events, stroke or cerebrovascular death. In cases of mortality, data were collected by an authorised local physician from the hospital or the death certificate and were evaluated by the TLGS outcome committee. We also examined the composite outcome including both CVD and all-cause mortality events. 


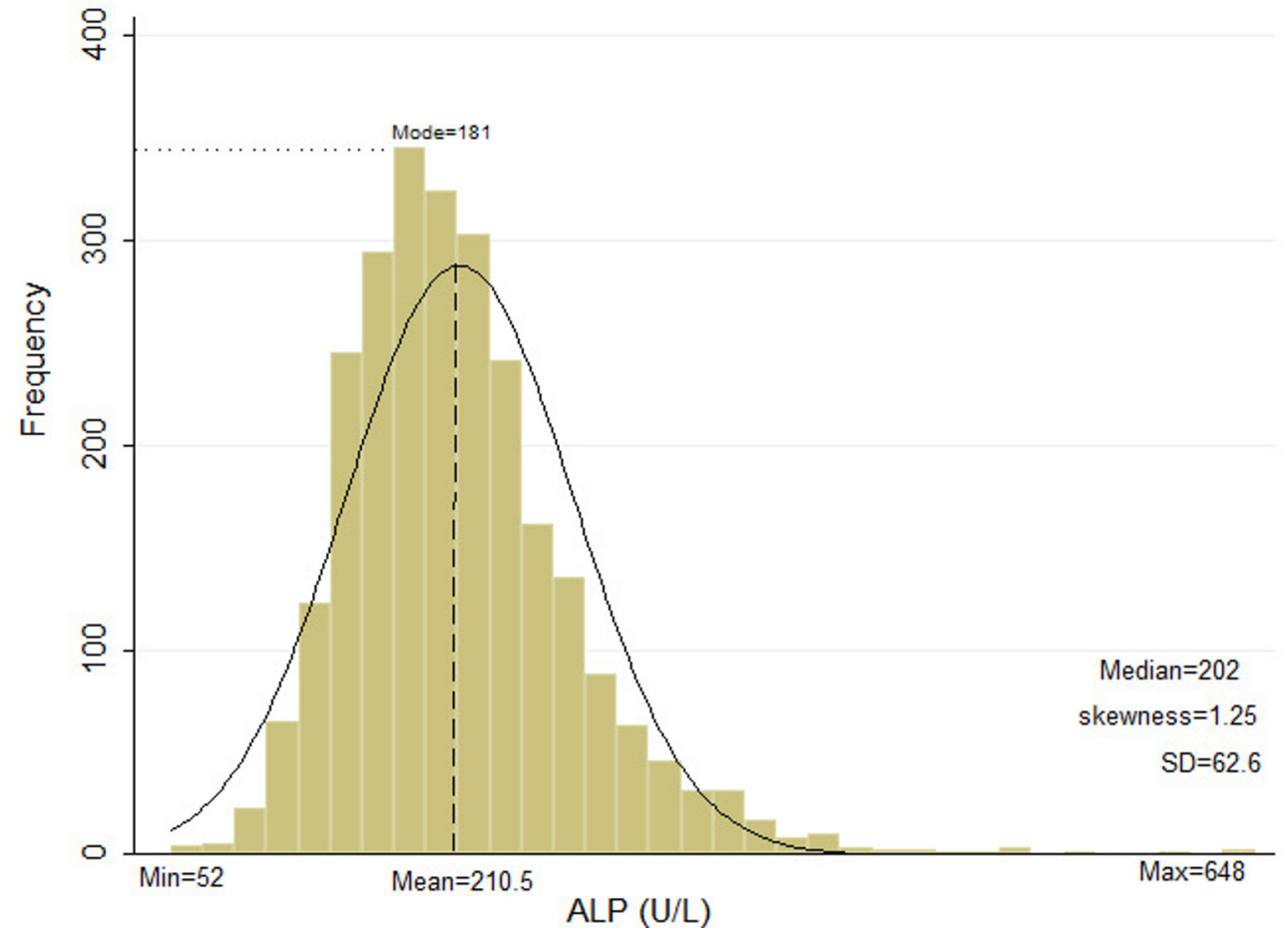

Figure 1 Distribution of baseline alkaline phosphatase (ALP) level among the study population. Max, maximum; Min, minimum.

\section{Definition of terms}

Type 2 diabetes was defined as $\mathrm{FPG} \geq 7 \mathrm{mmol} / \mathrm{L}$ or taking antihyperglycaemic drugs. Estimated glomerular filtration rate (eGFR) expressed as $\mathrm{mL} / \mathrm{min} / 1.73 \mathrm{~m}^{2}$ was estimated using the abbreviated prediction equation, and was calculated using the Chronic Kidney Disease Epidemiology Collaboration equation ${ }^{19}$ as follows:

$\mathrm{eGFR}=141 \times \min \quad\left(\mathrm{Scr} /{ }_{K}, \quad 1\right)^{a} \times \max \quad\left(\mathrm{Scr} /{ }_{K}\right.$, $1)^{-1.209} \times 0.993^{\text {age }} \times 1.018$ (if female) $\times 1.159$ (if black), where Scr is serum creatinine $(\mathrm{mg} / \mathrm{dL}), K$ is 0.7 for women and 0.9 for men, $a$ is -0.329 for women and -0.411 for men, min indicates the minimum of $\mathrm{Scr} /{ }_{K}$ or 1 and max indicates the maximum of $\mathrm{Scr} /{ }_{K}$ or 1 . CKD was defined as eGFR of $<60 \mathrm{~mL} / \mathrm{min}$ per $1.73 \mathrm{~m}^{2} .^{20}$

Hypertension was defined as either systolic blood pressure $(\mathrm{SBP}) \geq 140 \mathrm{~mm} \mathrm{Hg}$ or diastolic blood pressure (DBP) $\geq 90 \mathrm{~mm} \mathrm{Hg}$ or antihypertensive drug treatment. Smoking status was defined as current usage of any kind of tobacco. Family history of premature CVD reflected prior diagnosis of CVD in female first-degree relatives, aged $<65$ years or male first-degree relatives $<55$ years. Educational status was categorised into three levels: less than 6 years, 6-12 years and $\geq 12$ years of formal education.

\section{Statistical analysis}

Mean (SD) and frequency (\%) were reported for continuous and categorical variables, respectively. The Student's t-test and $\chi^{2}$ test were used to assess the significance of associations for continuous and categorical variables, respectively.

Dose-response relationship between ALP levels and different outcomes was checked using the fractional polynomial spline method in the confounder adjusted model with four knots (at 25th, 50th, 75th and 95th percentiles). Cox's proportional hazard models were used to evaluate associations between 1-SD as well as tertiles of ALP levels with the risks of CHD stroke, CVD, all-cause mortality and composite outcome.

These associations were evaluated in two models: model 1 included age and sex, and in model 2, for those with CHD, stroke, CVD and composite outcome further adjustment was performed for BMI, WC, smoking, educational levels, family history of premature CVD, TC, TG/HDL-C ratio, lipid-lowering medications, diabetes, hypertension and CKD. Moreover, for those with all-cause mortality event as outcome, model 2 entailed adjustment for a panel of variables (TLGS risk factors) reported to be associated with this event among the Iranian population according to our previous study ${ }^{21}$; TLGS risk factors included age, gender, current smoking, education, hypertension, diabetes and family history of premature CVD.

To determine the cut-off value of ALP for predicting composite outcome, the Youden's index ${ }^{22}$ was calculated as $[\mathrm{Y}=$ sensitivity-(1-specificity) $]$, in which the maximum value was considered as an optimum ALP cut-point. Moreover, indicators of diagnostic performance, including sensitivity, specificity, positive and negative predictive value, positive likelihood ratio (sensitivity/(1-specificity)) and negative likelihood ratio ((1-sensitivity)/specificity) for the cut-off points derived for each outcome were also calculated.

To examine the improvement in prediction of different outcomes in the models containing ALP level, we applied the AIC, the discrimination ability of models (Harrell's 
Table 1 Baseline characteristics of the study population, stratified by development of incident CVD at follow-up: Tehran Lipid and Glucose Study (1999-2014)

\begin{tabular}{|c|c|c|c|c|}
\hline Mean (SD) or $\mathrm{n}(\%)$ & $\begin{array}{l}\text { Total population } \\
(\mathrm{n}=2578)\end{array}$ & $\begin{array}{l}\text { Without CVD events } \\
(\mathrm{n}=2158)\end{array}$ & $\begin{array}{l}\text { With CVD events } \\
(n=420)\end{array}$ & $\mathbf{P}$ values \\
\hline Age (year) & $50.06(11.85)$ & $48.76(11.71)$ & $56.78(10.17)$ & $<0.001$ \\
\hline Sex (men) & $1089(42.24)$ & $874(40.50)$ & $215(51.19)$ & $<0.001$ \\
\hline $\operatorname{BMI}\left(\mathrm{kg} / \mathrm{m}^{2}\right)$ & $28.61(4.33)$ & $28.60(4.34)$ & $28.66(4.33)$ & 0.792 \\
\hline WC $(\mathrm{cm})$ & $94.23(10.55)$ & $93.79(10.54)$ & $96.49(10.36)$ & $<0.001$ \\
\hline $\mathrm{SBP}(\mathrm{mm} \mathrm{Hg})$ & $126.65(20.46)$ & $124.81(19.37)$ & $136.07(23.156)$ & $<0.001$ \\
\hline $\mathrm{DBP}(\mathrm{mm} \mathrm{Hg})$ & $81.57(10.87)$ & $81.08(10.48)$ & $84.12(12.41)$ & $<0.001$ \\
\hline $\mathrm{FPG}(\mathrm{mmol} / \mathrm{L})$ & $6.03(2.41)$ & $5.84(2.20)$ & $6.95(3.15)$ & $<0.001$ \\
\hline Total cholesterol (mmol/L) & $6.56(1.10)$ & $6.50(1.09)$ & $6.85(1.10)$ & $<0.001$ \\
\hline Triglycerides (mmol/L) & $3.05(1.69)$ & $3.01(1.67)$ & $3.26(1.80)$ & 0.006 \\
\hline TG/HDL-C & $3.35(2.87)$ & $3.31(2.88)$ & $3.55(2.86)$ & 0.113 \\
\hline eGFR $\left(\mathrm{mL} / \mathrm{min} / 1.73 \mathrm{~m}^{2}\right)$ & $66.36(11.24)$ & $66.92(11.24)$ & $63.49(10.82)$ & $<0.001$ \\
\hline ALP (IU/L) & $210.52(62.61)$ & $207.52(60.85)$ & $225.93(69.02)$ & $<0.001$ \\
\hline Family history of premature CVD & $461(17.88)$ & $374(17.33)$ & $87(20.71)$ & 0.098 \\
\hline Current smoker & $400(15.52)$ & $313(14.50)$ & $87(20.71)$ & 0.001 \\
\hline Education & & & & $<0.001$ \\
\hline$<6$ years & $1259(48.84)$ & $1000(46.34)$ & $259(61.67)$ & \\
\hline $6-12$ years & $1040(40.34)$ & $909(42.12)$ & $131(31.19)$ & \\
\hline$>12$ years & $279(10.82)$ & $249(11.54)$ & $30(7.14)$ & \\
\hline Lipid-lowering medications & 179 (6.94) & $136(6.30)$ & $43(10.24)$ & 0.004 \\
\hline Hypertension & $889(34.48)$ & $669(31)$ & $220(52.38)$ & $<0.001$ \\
\hline Diabetes & $401(15.55)$ & $271(12.56)$ & $130(30.95)$ & $<0.001$ \\
\hline CKD & 721 (27.97) & $567(26.27)$ & 154 (36.67) & $<0.001$ \\
\hline
\end{tabular}

ALP, alkaline phosphatase; BMI, body mass index; CKD, chronic kidney disease; CVD, cardiovascular disease; DBP, diastolic blood pressure; eGFR, estimated glomerular filtration rate (as calculated using the CKD Epidemiology Collaboration equation); FPG, fasting plasma glucose; HDL-C, high-density lipoprotein cholesterol; SBP, systolic blood pressure; TG, triglycerides; WC, waist circumference.

C-index) and the reclassification method using NRI ${ }^{14} 15$ for censored time-to-event data given as below:

AIC, was computed as $-2 *(\log$-likelihood $)+2 *($ number of estimated parameters) as a statistical estimate of the trade-off between the likelihood of a model against its complexity; the difference of AIC $\geq 10$ was considered significant.

To investigate the change in C-index after adding ALP information for prediction of CHD, stroke, CVD and composite outcome, we added ALP to a model based on risk factors included in the Framingham CVD Risk Score, ${ }^{23}$ which has been validated among Iranian populations. ${ }^{24}$ Regarding all-cause mortality, the change in C-index after adding ALP to a model based on TLGS risk factors was investigated.

We applied NRI as a criterion to evaluate the ability of adding ALP values to improve CHD, stroke, CVD and the composite outcome risk prediction over and beyond models containing Framingham risk factors, as well as the ability for improving prediction of the risk of all-cause mortality outcome over and beyond TLGS risk factors. The NRI measures the difference in discrimination slopes between models with and without a new variable such as ALP in our analysis ${ }^{15}$; significance of NRI was tested with bootstrap resampling. ${ }^{25}$

In a sensitivity analysis, the multiplicative interactions between ALP levels and all named confounders such as lipid panels (eg, ALP $\times$ TC and ALP $\times$ TG/HDL-C) were checked in a multivariate adjusted model for the composite outcome. Since a statistically significant interaction was found only for TC ( $\mathrm{p}$ value $=0.001$ ), the spline method was used to show the dose-response relationship between TC and the composite outcome for levels of ALP above and below the derived cut-off value.

Statistical significance was defined as a $\mathrm{p}$ value $<0.05$ in a two-sided manner. Statistical analyses were conducted using Stata V.12.0 (Stata Corp LP, College Station, Texas, USA).

\section{RESULTS}

The study included 2578 (men=1089) participants with a mean age of $50.06 \pm 11.85$ years at baseline, mean ALP level was 210.52 (SD=62.61) IU/L (median 202; IQR 73, 
figure 1). Baseline characteristics among the total population and based on occurrence of incident CVD are shown in table 1. Among the total population, the prevalence of current smoking, hypertension, diabetes and CKD were $15.52 \%, 34.48 \%, 15.55 \%$ and $27.97 \%$, respectively. Participants who developed CVD were significantly older, had higher levels of WC, SBP, DBP, FPG, TC, TG and ALP as well as higher prevalence of hypertension, diabetes, CKD, usage of lipid-lowering drugs, and were more likely to be smokers and less likely to be educated; however, there was no difference between groups regarding levels of BMI, TG/HDL-C and family history of premature CVD events.

During our study period, 369, 68, 420, 170 and 495 participants experienced CHD, stroke, CVD, all-cause mortality (17 participants had both CHD and stroke events) and composite outcome. Overall, $47.65 \%(\mathrm{n}=81)$ of all-cause mortality events were related to CVD. The median (IQR) follow-up duration was 11.3 (2.19) years.

Figure 2 shows dose-response relationship between risk of CHD, stroke, GVD, all-cause mortality and the composite outcome and ALP levels. Using the regression cubic spline model, a linear association was seen between serum ALP level and the 11-year risk of developing different outcomes.

Table 2 shows the adjusted HRs and 95\% CI of CHD, stroke, CVD, all-cause mortality and composite outcome for tertiles and per 1-SD increase in ALP in different models. Accordingly, HRs (95\% CI) for CHD events in the second and third tertiles of ALP levels after adjustment for important traditional risk factors (model 2) were 1.33 (1.02 to 1.73 ) and 1.29 (0.98 to 1.69 ), respectively. Regarding stroke events, the corresponding HRs (95\% CI) were 1.32 (0.66 to 2.61) and 1.88 (1.00 to 3.61), for second and third ALP tertiles, respectively. Moreover, mentioned tertiles were significantly associated with increased risk of CVD events with the adjusted HRs of 1.33 (1.03-1.71) and 1.30 (1.01-1.68), respectively. Furthermore, each 1-SD increase in ALP level was associated with $11 \%, 20 \%$ and $10 \%$ higher risk of CHD, stroke and CVD events in the fully adjusted model, respectively, with the risk calculated for stroke being marginally significant (HRs (95\% CI), 1.20 (0.97 to 1.49), $\mathrm{p}=0.058$ ).

Regarding all-cause mortality HRs of the second and third ALP tertiles, after adjustment for age and sex (model 1) were 1.13 (95\% CI 0.75 to 1.70$)$ and 1.64 (95\% CI 1.10 to 2.43), respectively. However, after further adjustment for TLGS risk factors (model 2), the aforementioned ALP tertiles were associated with $11 \%$ and $32 \%$ higher risks of events, values which did not reach statistical significance. Moreover, each 1-SD increase in ALP level was significantly associated with all-cause mortality events in both models 1 and 2 (HRs (95\% CI) were $1.25(1.10-1.42)$ and 1.16 (1.01-1.33), respectively). Finally, there was a significant association between ALP and the composite outcome with an adjusted HR (95\% CI) of 1.30 (1.03 to 1.63) and 1.27 (1.00 to 1.61) for the second and third tertiles, respectively, as well as 1.11 (1.02 to 1.21) for each 1-SD increase of ALP level in the fully adjusted model.
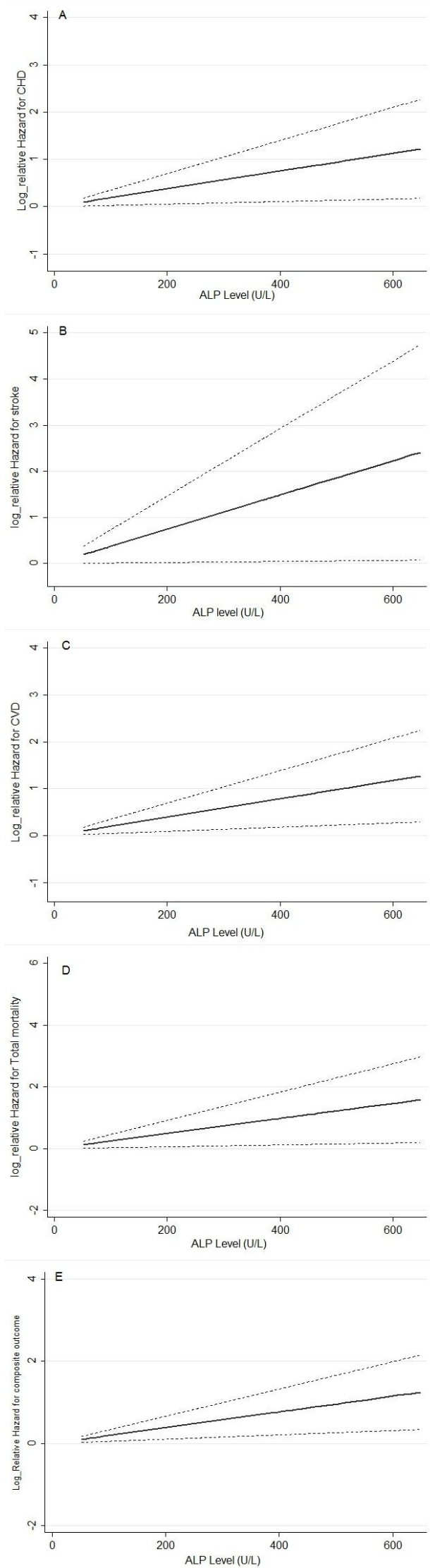

Figure 2 Regression cubic spline model of the association between alkaline phosphatase (ALP) and (A) coronary heart disease (CHD), (B) stroke, $(C)$ cardiovascular disease (CVD), (D) all-cause mortality and (E) the composite outcome. 
Table 2 Adjusted HR $(95 \% \mathrm{Cl})$ for cardiovascular disease, coronary heart disease, stroke and all-cause mortality events by tertiles and per 1-SD change of alkaline phosphatase: Tehran Lipid and Glucose Study (1999-2014)

\begin{tabular}{|c|c|c|c|c|c|}
\hline $\begin{array}{l}\text { Alkaline phosphatase } \\
\text { tertiles }\end{array}$ & First $(n=845)$ & Second $(n=858)$ & Third $(n=875)$ & & \\
\hline $\begin{array}{l}\text { Alkaline phosphatase level } \\
\text { (IU/L) }\end{array}$ & $<179$ & 179-226 & $>226$ & $\begin{array}{l}P \text { for } \\
\text { trend }\end{array}$ & $\begin{array}{l}\text { 1-SD increase in } \\
\text { ALP }\end{array}$ \\
\hline \multicolumn{6}{|l|}{ Coronary heart disease } \\
\hline $\mathrm{n}$ & 93 & 138 & 138 & & \\
\hline $\begin{array}{l}\text { Rate per } 1000 \text { person- } \\
\text { years }\end{array}$ & $9.7(7.92-11.89)$ & $14.94(12.64-17.65)$ & $17.57(14.87-20.77)$ & & \\
\hline Model $1^{*}$ & 1.00 & $1.39(1.06-1.81)$ & $1.59(1.22-2.08)$ & 0.001 & $1.21(1.10-1.33)$ \\
\hline Model $2 \dagger$ & 1.00 & $1.33(1.02-1.73)$ & $1.29(0.98-1.69)$ & 0.078 & $1.11(1.01-1.22)$ \\
\hline \multicolumn{6}{|l|}{ Stroke } \\
\hline$n$ & 14 & 22 & 32 & & \\
\hline $\begin{array}{l}\text { Rate per } 1000 \text { person- } \\
\text { years }\end{array}$ & $1.4(0.83-2.37)$ & $2.2(1.47-3.41)$ & $3.85(2.72-5.43)$ & & \\
\hline Model $1^{*}$ & 1.00 & $1.34(0.68-2.64)$ & $2.10(1.11-4.00)$ & 0.017 & $1.24(1.01-1.52)$ \\
\hline Model 2† & 1.00 & $1.32(0.66-2.61)$ & $1.88(1.00-3.61)$ & 0.050 & $1.20(0.97-1.49) \ddagger$ \\
\hline \multicolumn{6}{|l|}{ Cardiovascular disease } \\
\hline $\mathrm{n}$ & 104 & 155 & 161 & & \\
\hline $\begin{array}{l}\text { Rate per } 1000 \text { person- } \\
\text { years }\end{array}$ & $10.90(8.9-13.21)$ & $16.84(14.39-19.71)$ & 20.63 (17.68-24.08) & & \\
\hline Model 1* & 1.00 & $1.38(1.08-1.78)$ & $1.63(1.27-2.09)$ & $<0.001$ & $1.21(1.11-1.33)$ \\
\hline Model $2 \dagger$ & 1.00 & $1.33(1.03-1.71)$ & $1.30(1.01-1.68)$ & 0.052 & $1.10(1.01-1.21)$ \\
\hline \multicolumn{6}{|l|}{ All-cause mortality events } \\
\hline$n$ & 40 & 55 & 75 & & \\
\hline $\begin{array}{l}\text { Rate per } 1000 \text { person- } \\
\text { years }\end{array}$ & $4.00(2.93-5.45)$ & $5.58(4.28-7.27)$ & $8.93(7.12-11.20)$ & & \\
\hline Model $1^{*}$ & 1.00 & $1.13(0.75-1.70)$ & $1.64(1.10-2.43)$ & 0.009 & $1.25(1.10-1.42)$ \\
\hline Model $2 \S$ & 1.00 & $1.11(0.73-1.67)$ & $1.32(0.90-2.00)$ & 0.129 & $1.16(1.01-1.33)$ \\
\hline \multicolumn{6}{|l|}{ Composite outcome } \\
\hline $\mathrm{n}$ & 125 & 182 & 188 & & \\
\hline $\begin{array}{l}\text { Rate per } 1000 \text { person- } \\
\text { years }\end{array}$ & $13.1(11.0-15.6)$ & $19.8(17.1-22.9)$ & $24.1(20.9-27.8)$ & & \\
\hline Model $1^{*}$ & 1.00 & $1.33(1.06-1.67)$ & $1.52(1.21-1.92)$ & $<0.001$ & $1.20(1.11-1.31)$ \\
\hline Model $2 \dagger$ & 1.00 & $1.30(1.03-1.63)$ & $1.27(1.00-1.61)$ & 0.06 & $1.11(1.02-1.21)$ \\
\hline
\end{tabular}

*Model 1: adjusted for age and sex.

†Model 2: adjustment for model 1 plus body mass index, waist circumferences, smoking, educational levels, family history of premature cardiovascular disease, total cholesterol, triglycerides/high-density lipoprotein cholesterol ratio, lipid-lowering medications, diabetes, hypertension and chronic kidney disease.

$\neq \mathrm{P}=0.058$

$\S$ Model 2: Adjustment for model 1 plus Tehran Lipid and Glucose Study risk factors (ie, age, sex, current smoking, education, hypertension, diabetes, family history of premature CVD).

ALP, alkaline phosphatase.

In this study, the optimal cut-off level of ALP value according to the Youden's index for the composite outcome was $199 \mathrm{IU} / \mathrm{L}$. The diagnostic performance of the derived cut-off level of ALP was examined for each outcome (see online supplementary table 1). Table 3 shows the adjusted HR (95\% CI) of CVD, CHD, stroke, all-cause mortality events and the composite outcome for the derived ALP cut-off point. Accordingly, the risks of all outcomes were higher in those with ALP $\geq 199$ IU/L level when adjusted with age and sex. Moreover, considering covariates in model 2, significant risks of $80 \%, 26 \%, 43 \%$ and $26 \%$ were shown for incident stroke, CVD, all-cause mortality and the composite outcome.

Risk discrimination, goodness of fit and reclassification index, with and without adding ALP values to the Framingham and TLGS risk prediction models are shown in 
Table 3 Adjusted HR $(95 \% \mathrm{Cl})$ for cardiovascular disease, coronary heart disease, stroke, all-cause mortality events and the composite outcome for the derived ALP cut-off point (ALP $\geq 199 \mathrm{IU} / \mathrm{L})^{\star}$ :Tehran Lipid and Glucose Study (1999-2014)

\begin{tabular}{|c|c|c|}
\hline \multirow{2}{*}{ Outcomes } & \multicolumn{2}{|c|}{ Alkaline phosphatase (IU/L) } \\
\hline & $<199(n=1223)$ & $\geq 199$ ( $n=1355)$ \\
\hline \multicolumn{3}{|l|}{ Coronary heart disease } \\
\hline $\mathrm{n}$ & 139 & 230 \\
\hline $\begin{array}{l}\text { Rate per } 1000 \text { person- } \\
\text { years }\end{array}$ & $10.8(9.2-12.8)$ & $16.6(14.6-18.9)$ \\
\hline Model $1 \dagger$ & 1.00 & $1.39(1.13-1.72)$ \\
\hline Model $2 \ddagger$ & 1.00 & $1.19(0.96-1.48)$ \\
\hline \multicolumn{3}{|l|}{ Stroke } \\
\hline $\mathrm{n}$ & 19 & 49 \\
\hline $\begin{array}{l}\text { Rate per } 1000 \text { person- } \\
\text { years }\end{array}$ & $1.4(0.9-2.2)$ & $3.3(2.5-4.4)$ \\
\hline Model $1 \dagger$ & 1.00 & $1.94(1.13-3.34)$ \\
\hline Model $2 \ddagger$ & 1.00 & $1.80(1.04-3.1)$ \\
\hline \multicolumn{3}{|l|}{ Cardiovascular disease } \\
\hline $\mathrm{n}$ & 153 & 267 \\
\hline $\begin{array}{l}\text { Rate per } 1000 \text { person- } \\
\text { years }\end{array}$ & $12.0(10.2-14.0)$ & $19.4(17.2-21.9)$ \\
\hline Model $1 \dagger$ & 1.00 & $1.45(1.19-1.78)$ \\
\hline Model $2 \ddagger$ & 1.00 & $1.26(1.02-1.54)$ \\
\hline \multicolumn{3}{|l|}{ All-cause mortality events } \\
\hline $\mathrm{n}$ & 52 & 118 \\
\hline $\begin{array}{l}\text { Rate per } 1000 \text { person- } \\
\text { years }\end{array}$ & $3.9(2.9-5.1)$ & $8.0(6.7-9.6)$ \\
\hline Model 1† & 1.00 & $1.64(1.18-2.29)$ \\
\hline Model $2 \S$ & 1.00 & $1.43(1.02-2.00)$ \\
\hline \multicolumn{3}{|l|}{ Composite outcome } \\
\hline $\mathrm{n}$ & 179 & 316 \\
\hline $\begin{array}{l}\text { Rate per } 1000 \text { person- } \\
\text { years }\end{array}$ & $14.0(12.1-16.2)$ & $22.9(20.6-25.6)$ \\
\hline Model $1 \dagger$ & 1.00 & $1.43(1.19-1.72)$ \\
\hline Model $2 \ddagger$ & 1.00 & $1.26(1.04-1.52)$ \\
\hline
\end{tabular}

${ }^{*}$ Cut-off based on Youden's index for composite outcome. †Model 1: adjusted for age and sex.

¥Model 2: adjustment for model 1 plus body mass index, waist circumferences, smoking, educational levels, family history of premature cardiovascular disease, total cholesterol, triglycerides/high-density lipoprotein cholesterol ratio, lipid-lowering medications, diabetes, hypertension and chronic kidney disease.

§Model 2: adjustment for for model 1 plus Tehran Lipid and Glucose Study (TLGS) risk factors (ie, age, sex, current smoking, education, hypertension, diabetes, family history of premature CVD).

ALP, alkaline phosphatase.

table 4. CHD and CVD risk prediction models containing Framingham risk factors showed a C-index of 0.7380 (0.709-0.766) and $0.7462(0.717-0.774)$, respectively, and after adding ALP values, the C-indexes reached 0.7384 $(0.710-0.765)$ and $0.7469 \quad(0.724-0.767)$, showing an increase of 0.0004 and 0.0007 , respectively, which were not statistically significant. In addition, there was no significant improvement in the classification of participants for CHD and CVD events applying NRI $(0.79(-0.29-0.45)$ and 0.72 (-0.39-1.36), respectively) and no significant differences in
AIC were seen among these two models. Regarding all-cause mortality and the composite outcome, similar to CVD events, after adding information of ALP, no improvement was observed in the predictive ability of the models considering the levels of AIC, the C-index and NRI. Since, the association between ALP level and stroke events was marginally significant, we did not assess the added value of ALP level to predict this outcome.

According to significant interaction between TC and ALP for the composite outcome, the association of ALP $\geq 199$ versus $<199 \mathrm{IU} / \mathrm{L}$ across the entire range of $\mathrm{TC}$ was examined. Accordingly, for the ALP value $<199 \mathrm{IU} / \mathrm{L}$, the association remained linear across the entire range of TC levels, while for the ALP value $\geq 199 \mathrm{IU} / \mathrm{L}$, this association showed a non- linear shape (see online supplementary figure 1).

\section{DISCUSSION}

In this prospective population-based study, we found an association independent of established cardiovascular risk factors between baseline ALP level and future CVD events over more than a decade of follow-up. In fact, ALP level $>179 \mathrm{IU} / \mathrm{L}$ was associated with over $30 \%$ higher risk of CVD (including CHD and stroke events), independent of several established traditional CVD risk factors. However, our study showed that adding ALP values to conventional CVD risk factors does not lead to improvement in their ability to predict CVD events. In addition, our analyses showed that each $62.61 \mathrm{IU} / \mathrm{L}$ (1-SD) increase in ALP level was associated with $16 \%$ greater risk of all-cause mortality events, independent of a large set of covariates; however, as mentioned earlier regarding CVD events, adding ALP values does not improve the prediction power for mortality risk.

Association between serum ALP and increased risk of $\mathrm{CHD},{ }^{10}$ stroke, ${ }^{102627} \mathrm{CVD}^{81028}$ as well as all-cause mortality ${ }^{9}{ }^{28-31}$ has been addressed in some previous studies; nevertheless, direct comparison of our results with theirs is not simple due to different strategies for inclusion of the study populations and especially different adjustments that were considered in their multivariate analysis. Finally, they used different approaches in presenting their results.

To the best of our knowledge, the association between elevated ALP levels and CVD (including both CHD and stroke events) in general populations has been studied in five population-based studies conducted in the USA, Europe and Japan. Among studies that focused on CVD or CVD death, the HRs (95\% CI) in fully adjusted models were 1.27 (0.98 to 1.65$)$ in the Third National Health and Nutrition Examination Survey study ${ }^{28}$ (comparing the third vs first tertile of ALP), 1.09 (1.01 to 1.18) in the British Regional Heart Study (BRHS) ${ }^{10}$ (per 1-SD increase in ALP) and 1.24 (1.05 to 1.45) in the Prevention of Renal and Vascular End-stage Disease study ${ }^{8}$ (comparing fifth vs first-fourth ALP quintiles). In addition, three studies have examined this association for stroke events and documented the following results in 


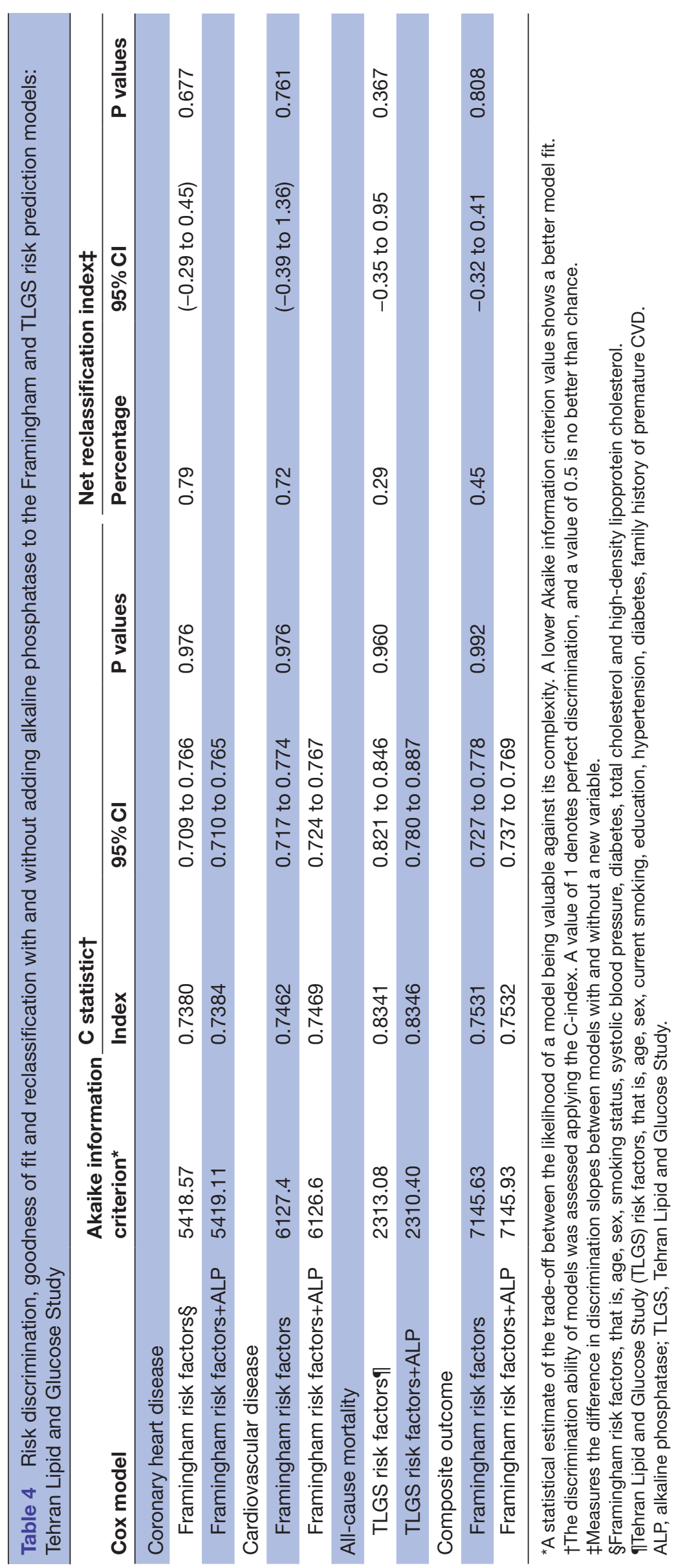


fully adjusted models: HR 1.09 (0.96 to 1.25) per 1-SD increase in log-ALP level in the BRHS study, ${ }^{10}$ HR 1.79 (1.20 to 2.68) in men and 1.29 (0.85 to 1.96 ) in women for ALP fifth quintile versus third quintile in the Circulatory Risk in Communities Study ${ }^{26}$ and Relative Risk (RR) is 1.20 (1.04-1.39) per each 1-SD increment of ALP level in the Rotterdam Study. ${ }^{27}$ In the current study, in the multivariate analysis, each 1-SD increase in ALP level was associated with approximately 20\% higher risk of stroke. Moreover, compared with those with ALP levels $<179 \mathrm{IU} / \mathrm{L}$, ALP values $>226 \mathrm{IU} / \mathrm{L}$ increased this risk to over $80 \%$. Only one study has assessed the association between higher ALP levels and the risk of CHD among a general population and the results showed each 1-SD increase in ALP level was associated with $15 \%$ higher risk in the fully adjusted model ${ }^{10}$ results consistent with ours that showed an $11 \%$ higher risk. Finally, a recent meta-analysis performed by Kunutsor et $a l^{11}$ has summarised these results and shown that the multivariate relative risk for CVD events per 1-SD change in baseline ALP was 1.09 (1.02 to 1.16), a finding comparable with our results (HR (95\% CI) 1.10 (1.01 to 1.21)). Despite the independent association between ALP levels and CHD, stroke and CVD in our study, we found no added value for ALP in prediction of CVD events, results similar to those reported in the Kunutsor et al study. ${ }^{8}$ Moreover, the association between ALP level and mortality in general populations has been addressed in studies conducted by Tonelli et $a l^{28}$ Wannamethee et $a l^{10}$ Abramowitz et al, ${ }^{9}$ Filipowicz et $a l^{30}$ Kohler et $a l^{32}$ Fulks et $a l^{29}$ and Bates $e t$ $a l l^{31}$ all of which were conducted in the USA and Europe. Accordingly, a recent meta-analysis ${ }^{12}$ showed that the multivariate relative risk $(95 \% \mathrm{CI})$ for all-cause mortality in the top, compared with the bottom third of ALP level was 1.38 ( 1.17 to 1.63$)$, a value comparable to those derived in our data analysis (1.32 (0.90 to 2.00$))$. Similar to CVD events, we found no improvement in prediction of all-cause mortality events after adding ALP data to the known risk factors, an issue not addressed in the studies mentioned above.

Previously, it has been shown that among maintenance haemodialysis patients, there are robust data regarding a consistent association of increased ALP level with all-cause and cardiovascular mortality, ${ }^{33-36}$ as well as coronary artery calcification $^{37}$; accordingly, a serum ALP $>120$ IU/L was associated with higher risk of outcomes across different subgroups of patients, after controlling for nutrition, inflammation, mineral, serum Parathyroid Hormone (PTH) and liver enzymes. In the current study, among a general population, the derived cut-off point of 199 showed significant risk for GVD, stroke and all-cause mortality as well as the composite outcome.

Among general populations, several mechanisms have been proposed for increased cardiovascular and mortality risks in people with elevated ALP levels. There is some evidence that low levels of vitamin $\mathrm{D}$ are associated with elevated ALP and CVD mortality, as shown in a nested case-control study within our cohort, 25-OH-D levels $<10 \mathrm{ng} / \mathrm{mL}$ were associated with an approximately threefold higher risk of CVD after adjustment for conventional CVD risk factors ${ }^{38}$; however, data on vitamin D levels were not available in our study, although, considering that the prevalence of severe vitamin D deficiency among Tehranian adults is reported to be $<10 \%,{ }^{39}$ it might not be an important confounder in our study. Moreover, the association between ALP and CVD might be through inflammation as ALP is an acute phase reactant and its activity is correlated with plasma $\mathrm{C}$ reactive protein (CRP) levels. ${ }^{40}$ We did not have data on CRP levels so this possibility could not be investigated. However, in a nested case-control study of 385 individuals (126 cases, free of cardiovascular disease at baseline, and 259 matched controls) among our population, the level of CRP did not predict the incidence of CVD, independent of traditional CVD risk factors. ${ }^{41}$ In addition, in some previous studies, ALP showed an independent association with $\mathrm{CVD}^{10}$ and all-cause mortality, ${ }^{28}$ after adjustment for conventional CVD risk factors and CRP. The association between elevated ALP and CVD might be due to liver disease, primarily non-alcoholic fatty liver disease (NAFLD), ${ }^{42}$ a condition which is a common cause of elevated liver enzymes, and is associated with insulin resistance, type 2 diabetes and obesity. Moreover, NAFLD confers higher risk of CVD, independent of traditional cardiovascular risk factors and metabolic syndrome. ${ }^{43}$ Results of a meta-analysis from Iran ${ }^{44}$ demonstrated the prevalence of NAFLD to be $33.9 \%$; hence, considering the high prevalence of NAFLD among the Iranian population, the role of subclinical liver disease in the association between ALP and CVD cannot be overlooked. Other possible mechanisms for higher cardiovascular and mortality risks associated with higher ALP levels have been suggested, including vascular calcification, endothelial dysfunction, inactivation of inorganic pyrophosphates (a process that can increase vascular calcification) as well as inflammation and oxidative stress. ${ }^{72845}$

The notable strengths of our study include a large sample size with long-term follow-up duration, adequate adjustment for potential confounders and reliable measurements of different covariates as well as assessing different outcomes including CHD, stroke, CVD and all-cause mortality. We also extended findings of a prior research showing no added value of ALP level in prediction of different outcomes; moreover, we presented the cut-off value of ALP level for prediction of composite CVD and mortality events. Of the limitations that should be noted, first, some evidence suggests that ALP values in individuals can fluctuate over time ${ }^{46}$ and repeated ALP measurements were not available in our study. Second, data on other confounders, that is, PTH, CRP, vitamin $\mathrm{D}$ and liver enzymes including alanine and aspartate transaminase were also unavailable. Third, we did not have nutritional information. Finally, as this study was conducted on indivuduals of Persian ancestry, its generalisability to other populations is not possible. 
In conclusion, the current evidence from an Iranian population shows an independent association between ALP level and the risk of CHD, stroke, CVD, all-cause mortality and the composite outcome, although when the ALP data were added to the conventional risk prediction models, it did not improve their ability for prediction of these outcomes.

\section{Author affiliations}

${ }^{1}$ Clinical Research Development Unit, Sayad Shirazi Hospital, Golestan university of Medical Sciences, Gorgan, Golestan, Iran

${ }^{2}$ Prevention of Metabolic Disorders Research Center, Research Institute for Endocrine Sciences, Shahid Beheshti University of Medical Sciences, Tehran, Iran ${ }^{3}$ Department of Internal Medicine, School of Medicine, Shahid Beheshti University of Medical Sciences, Tehran, Iran

${ }^{4}$ Occupational Sleep Research Center, Baharloo Hospital, Tehran University of Medical Sciences, Tehran, Iran

${ }^{5}$ Endocrine Research Center, Research Institute for Endocrine Sciences, Shahid Beheshti University of Medical Sciences, Tehran, Iran

Acknowledgements We would like to express our appreciation to the research team members and to TLGS participants for their enthusiastic support. The authors also wish to acknowledge Ms Niloofar Shiva for critical editing of English grammar and syntax of the manuscript.

Contributors Conception and design, or analysis and interpretation of data, or both: FH, MK, MRR, SAk and SAs. Drafting the article or revising it critically for important intellectual content: FH, MK, MRR, SAk, SAs and FA. All authors contributed to the writing of the paper, and read and approved the final manuscript.

Funding The authors have not declared a specific grant for this research from any funding agency in the public, commercial or not-for-profit sectors.

Competing interests None declared.

Patient consent Obtained.

Ethics approval Ethics committee of the Research Institute for Endocrine Sciences, Shahid Beheshti University of Medical Sciences approved the design of the TLGS.

Provenance and peer review Not commissioned; externally peer reviewed.

Data sharing statement Any additional data will be available upon the journal request.

Open access This is an open access article distributed in accordance with the Creative Commons Attribution Non Commercial (CC BY-NC 4.0) license, which permits others to distribute, remix, adapt, build upon this work non-commercially, and license their derivative works on different terms, provided the original work is properly cited, appropriate credit is given, any changes made indicated, and the use is non-commercial. See: $\mathrm{http}: / /$ creativecommons.org/licenses/by-nc/4.0/.

\section{REFERENCES}

1. Murray CJL, Vos T, Lozano R, et al. Disability-adjusted life years (dalys) for 291 diseases and injuries in 21 regions, 1990-2010: A systematic analysis for the global burden of disease study 2010 . The Lancet 2012;380:2197-223.

2. Kochanek KD, Murphy SL, Xu J, et al. Deaths: final data for 2014 National vital statistics reports: from the Centers for Disease Control and Prevention, National Center for Health Statistics, National Vital Statistics System. 2016;65:1-122.

3. Khalili D, Sheikholeslami FH, Bakhtiyari M, et al. The incidence of coronary heart disease and the population attributable fraction of its risk factors in Tehran: a 10-year population-based cohort study. PLoS One 2014;9:e105804.

4. Hadaegh F, Harati H, Ghanbarian A, et al. Prevalence of coronary heart disease among Tehran adults: Tehran Lipid and Glucose Study, 2009.

5. Azizi F, Rahmani M, Emami H, et al. Cardiovascular risk factors in an Iranian urban population: Tehran Lipid and Glucose Study (Phase 1). Sozial- und Preventivmedizin/Social and Preventive Medicine 2002;47:408-26.
6. Libby P, Ridker PM, Maseri A. Inflammation and atherosclerosis. Circulation 2002;105:1135-43.

7. Haarhaus M, Brandenburg V, Kalantar-Zadeh K, et al. Alkaline phosphatase: a novel treatment target for cardiovascular disease in CKD. Nat Rev Nephrol 2017;13:429-42.

8. Kunutsor SK, Bakker SJ, Kootstra-Ros JE, et al. Serum alkaline phosphatase and risk of incident cardiovascular disease: Interrelationship with high sensitivity c-reactive protein. PLoS One 2015;10:e0132822

9. Abramowitz M, Muntner P, Coco M, et al. Serum alkaline phosphatase and phosphate and risk of mortality and hospitalization. Clin J Am Soc Nephrol 2010:5:1064-71.

10. Wannamethee SG, Sattar N, Papcosta O, et al. serum phosphate, and incident cardiovascular disease and total mortality in older men. Arteriosclerosis, thrombosis, and vascular biology. 2013;33:1070-6.

11. Kunutsor SK, Apekey TA, Khan H. Liver enzymes and risk of cardiovascular disease in the general population: a meta-analysis of prospective cohort studies. Atherosclerosis 2014;236:7-17.

12. Kunutsor SK, Apekey TA, Seddoh D, et al. Liver enzymes and risk of all-cause mortality in general populations: a systematic review and meta-analysis. Int J Epidemiol 2014;43:187-201.

13. Wong ND. Epidemiological studies of CHD and the evolution of preventive cardiology. Nat Rev Cardiol 2014;11:276-89.

14. Pencina MJ, D'Agostino RB, Steyerberg EW. Extensions of net reclassification improvement calculations to measure usefulness of new biomarkers. Stat Med 2011;30:11-21.

15. Pencina MJ, D'Agostino RB, D'Agostino RB, et al. Evaluating the added predictive ability of a new marker: from area under the ROC curve to reclassification and beyond. Stat Med 2008:27:157-72.

16. Azizi F, Ghanbarian A, Momenan AA, et al. Prevention of noncommunicable disease in a population in nutrition transition: Tehran Lipid and Glucose Study phase II. Trials 2009;10:5.

17. Khalili D, Mosavi-Jarrahi A, Eskandari F, et al. Evaluation of cause of deaths' validity using outcome measures from a prospective, population based cohort study in Tehran, Iran. PLoS One 2012; 7:e31427.

18. Organization WH. Cerebrovascular disorders: a clinical and research classification, 1978.

19. Levey AS, Stevens LA, Schmid $\mathrm{CH}$, et al. A new equation to estimate glomerular filtration rate. Ann Intern Med 2009;150:604-12.

20. Levey AS, Eckardt KU, Tsukamoto Y, et al. Definition and classification of chronic kidney disease: a position statement from Kidney Disease: Improving Global Outcomes (KDIGO). Kidney Int 2005;67:2089-100.

21. Sardarinia M, Akbarpour S, Lotfaliany $M$, et al. Risk factors for incidence of cardiovascular diseases and all-cause mortality in a middle eastern population over a decade follow-up: Tehran lipid and glucose study. PLoS One 2016;11:e0167623.

22. Youden WJ. Index for rating diagnostic tests. Cancer 1950;3:32-5.

23. D'Agostino RB, Vasan RS, Pencina MJ, et al. General cardiovascular risk profile for use in primary care. Circulation 2008;117:743-53.

24. Khalili D, Hadaegh F, Soori $\mathrm{H}$, et al. Clinical usefulness of the Framingham cardiovascular risk profile beyond its statistical performance: the Tehran Lipid and Glucose Study. Am J Epidemiol 2012;176:177-86.

25. Pepe MS, Feng Z, Gu JW. Comments on 'Evaluating the added predictive ability of a new marker: From area under the ROC curve to reclassification and beyond' by M. J. Pencina et al., Statistics in Medicine (DOI: 10.1002/sim.2929). Stat Med 2008:27:173-81.

26. Shimizu Y, Imano H, Ohira T, et al. Alkaline phosphatase and risk of stroke among Japanese: the Circulatory Risk in Communities Study (CIRCS). J Stroke Cerebrovasc Dis 2013;22:1046-55.

27. Wieberdink R, Koudstaal P, Hofman A, et al. Serum Liver Enzyme Levels And The Risk Of Stroke In The General Population: The Rotterdam Study. Cerebrovascular Diseases 2011;31:39.

28. Tonelli M, Curhan G, Pfeffer M, et al. Relation between alkaline phosphatase, serum phosphate, and all-cause or cardiovascular mortality. Circulation 2009;120:1784-92.

29 Fulks M, Stout RL, Dolan VF. Using liver enzymes as screening tests to predict mortality risk. J Insur Med 2008;40(3-4):191-203.

30. Filipowicz R, Greene T, Wei G, et al. Associations of serum skeletal alkaline phosphatase with elevated $\mathrm{C}$-reactive protein and mortality. Clin J Am Soc Nephrol 2013;8:26-32.

31. Bates CJ, Hamer M, Mishra GD. A Study of relationships between bone-related vitamins and minerals, related risk markers, and subsequent mortality in older british people: The national diet and nutrition survey of people aged 65 years and over. Osteoporos Int 2012;23:457-66. 
32. Koehler EM, Sanna D, Hansen BE, et al. Serum liver enzymes are associated with all-cause mortality in an elderly population. Liver Int 2014;34:296-304.

33. Regidor DL, Kovesdy CP, Mehrotra R, et al. Serum alkaline phosphatase predicts mortality among maintenance hemodialysis patients. J Am Soc Nephrol 2008;19:2193-203.

34. Kalantar-Zadeh K, Shah A, Duong U, et al. Kidney bone disease and mortality in CKD: revisiting the role of vitamin $\mathrm{D}$, calcimimetics, alkaline phosphatase, and minerals. Kidney Int Suppl 2010;78:S10-S21.

35. Sumida K, Molnar MZ, Potukuchi PK, et al. Prognostic significance of pre-end-stage renal disease serum alkaline phosphatase for post-end-stage renal disease mortality in late-stage chronic kidney disease patients transitioning to dialysis. Nephrology Dialysis Transplantation 2017;53:gfw412-73.

36. Molnar MZ, Kovesdy CP, Mucsi I, et al. Association of pre-kidney transplant markers of mineral and bone disorder with post-transplant outcomes. Clin J Am Soc Nephrol 2012;7:1859-71.

37. Shantouf R, Kovesdy CP, Kim Y, et al. Association of serum alkaline phosphatase with coronary artery calcification in maintenance hemodialysis patients. Clin J Am Soc Nephrol 2009;4:1106-14.

38. Hosseinpanah F, Yarjanli M, Sheikholeslami F, et al. Associations between vitamin D and cardiovascular outcomes; Tehran Lipid and Glucose Study. Atherosclerosis 2011;218:238-42.
39. Hashemipour S, Larijani B, Adibi H, et al. Vitamin D deficiency and causative factors in the population of Tehran. BMC Public Health 2004;4:38.

40. Webber M, Krishnan A, Thomas NG, et al. Association between serum alkaline phosphatase and C-reactive protein in the United States National Health and Nutrition Examination Survey 2005-2006. Clin Chem Lab Med 2010;48:167-73.

41. Tohidi M, Hadaegh $\mathrm{F}$, Harati $\mathrm{H}$, et al. C-reactive protein in risk prediction of cardiovascular outcomes: Tehran Lipid and Glucose Study. Int J Cardiol 2009;132:369-74.

42. Ndrepepa G. Alkaline phosphatase and cardiovascular disease. Journal of Laboratory and Precision Medicine 2017;2:83.

43. Patil R, Sood GK. Non-alcoholic fatty liver disease and cardiovascular risk. World J Gastrointest Pathophysiol 2017;8:51.

44. Moghaddasifar I, Lankarani KB, Moosazadeh M, et al. Prevalence of Non-alcoholic Fatty Liver Disease and Its Related Factors in Iran. Int J Organ Transplant Med 2016;7:149.

45. Lau WL, Kalantar-Zadeh K. Towards the revival of alkaline phosphatase for the management of bone disease, mortality and hip fractures. Nephrol Dial Transplant 2014;29:1450-2.

46. Lazo M, Selvin E, Clark JM. Brief communication: Clinical implications of short-term variability in liver function test resultsshortterm variability in liver function test results. Annals of internal medicine 2008;148:348-52. 